\section{Toward the Perfect}

At a meeting several years ago, Jim Armitage from Nebraska brought up an aphoristic dilemma: in the military world, "don't let the perfect become the enemy of the good" is an appropriate strategic approach. In the art world, however, "don't let the good become the enemy of the perfect" is more applicable. The question for clinicians, then, is whether we should operate in military or art world mode?

In a sense, clinical practice guidelines represent "the good." Because medical knowledge is imperfect in almost all areas, we recommend treatments that, even with the most innovative approaches tested in meticulously designed, high-powered trials, will not yield 100\% success. In many areas, "the perfect" is not even on a realistic horizon. Instead, guidelines committees such as the NCCN Pancreatic Cancer Panel must assess how the finding that adding erlotinib to gemcitabine increases 1 -year survival from $17 \%$ to $24 \%$ should be reflected in the treatment algorithm.

The 3 articles on Hodgkin disease/lymphoma (HD) in this issue bring the dilemma to the fore. Given the extraordinary success rates of $94 \%$ to $98 \%$ overall survival in early stage disease, documented in the article by Yahalom, and encouraging new salvage regimens that result in $80 \%$ to $90 \% 5$-year survival rates in advanced disease, as detailed by Advani et al., we can say that the good is approaching the perfect. But we still have steps to climb.

With high success rates come increasing options. For HD, those options include the chance to deal with a major roadblock to reaching the perfect goal: life-threatening and -diminishing side effects. The third paper in our trilogy, "Late Effects of Treatment for Hodgkin Lymphoma" by Friedman and Constine, should be required reading for students of HD. As these authors show, the risk of developing a second primary tumor for HD survivors is 7 to 18 times that of the general population. Therefore, investigating the treatment-related epidemiology of secondary malignant tumors is incredibly important. These studies, in turn, spawn a host of therapeutic options such as tailored radiation therapy doses and modified chemotherapy regimens, which then lead to achieving superb survival rates while diminishing deleterious sequelae. If we continue to strive for the perfect and as the science of chemoprevention matures, we will find new and more effective ways to curtail these secondary killers.

Thus, I believe that the perfect should still be our goal, and although lessening the events that shorten survival is incredibly important, it is not enough to reach the last pinnacle. A 95\% survival rate in HD is awesome, but 5 of 100 young people dying is not the ultimate goal. New agents and new approaches are necessary. "The good" is good, but I fear that if we accept this wonderful good instead of striving for the perfect, and stop placing our patients on clinical trials, we will never take that last magnificent step.

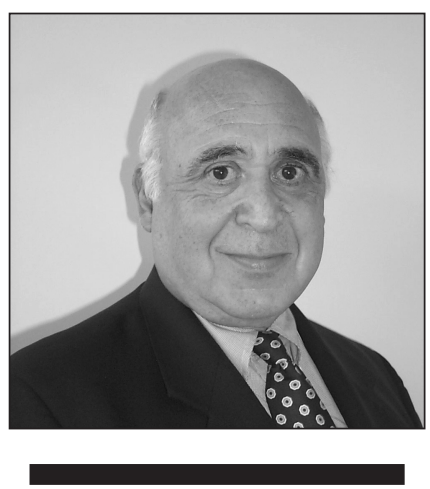

By Rodger J. Winn, MD

Rodger J. Winn is the Editor-inChief of JNCCN. He is Clinical Consultant at the National Quality Forum, and his past positions include Associate Professor of Clinical Medicine at The University of Texas M. D. Anderson Cancer Center. Dr. Winn received his medical degree from Jefferson Medical College of Philadelphia. His postgraduate training includes an internship and residency at Jefferson Medical College, and he also completed a medical oncology fellowship at Memorial Sloan-Kettering Cancer Center in New York. He is board certified in internal medicine and holds subspecialty certification in oncology. 